\title{
Clasificación digital de suelos a través de covariables ambientales de la cuenca del río Mixteco
}

\section{Digital soil classification trough environmental covariables of the Mixteco river watershed}

\author{
Gerardo Colín García ${ }^{1}$, Demetrio S. Fernández Reynoso², Mario R. Martínez Menez ${ }^{2}$, \\ José Donaldo Ríos Berber², Patricio Sánchez Guzmán², \\ Erasmo Rubio Granados ${ }^{2}$ y Laura Alicia Ibáñez Castillo ${ }^{3}$
}

\footnotetext{
${ }^{1}$ Posgrado de Hidrociencias, ${ }^{2}$ Programa de Edafología, Colegio de Postgraduados. Carretera México-Texcoco km 36.5. 56230 Montecillo, Texcoco, Estado de México, México.

† Autor responsable (mmario@colpos.mx)

${ }^{3}$ Departamento de Irrigación, Universidad Autónoma Chapingo. Carretera México-Texcoco km 38.5. 56230 Chapingo, Texcoco, Estado de México, México.
}

\section{RESUMEN}

La escasez de mapas con una distribución detallada de las unidades de suelo representa una limitante frecuente para la toma de decisiones, el modelado hidrológico y determinación del potencial productivo de un área. En este trabajo se aplicó la técnica de Mapeo Digital de Suelos (MDS), a través del Análisis de Componentes Principales (ACP), para obtener la distribución espacial de las unidades de suelo. El área de estudio corresponde a la cuenca del río Mixteco, con una superficie de 655903 ha, que se ubica en el estado de Oaxaca. Para el MDS se emplearon covariables ambientales del tipo topográficas, hidrológicas, climáticas y geológicas. Las covariables ambientales de mayor impacto, en la distribución espacial de las unidades de suelo, en orden de importancia fueron las geológicas, topográficas y climáticas. La clasificación digital se basó en 142 áreas de entrenamiento y 60 sitios para la validación de resultados, distribuidos aleatoriamente. En la cuenca, de acuerdo al Sistema de Clasificación WRB de la FAO (2014), a través del MDS se identificaron de manera satisfactoria (precisión global de 76.67\% y un Índice Kappa de 71.20) los siguientes grupos de suelos Cambisoles (35.8\%), Fluvisoles (7.16\%), Leptosoles (48.25\%), Regosoles $(8.00 \%)$ y Vertisoles (0.79\%). El MDS determinó satisfactoriamente la distribución espacial de las unidades de suelo en la cuenca e identificó las covariables ambientales de mayor influencia.
Palabras clave: mapeo digital de suelo, análisis de covarianza, unidades de suelo, Índice Kappa, región Mixteca.

\section{SUMMARY}

The lack of detailed maps of spatial distribution of soil units is a frequent constraint in decision-making processes, hydrological modeling, and productive assessment of an area. In this work, the Digital Soil Mapping (DSM) technique was applied, and Principal Component Analysis (PCA) was used to obtain the spatial distribution of dominant soil units in a watershed. The study area was the Mixteco watershed, which covers an area of 655903 ha, of the Mexican state of Oaxaca. For DSM, topographic, hydrological, climatic and geological environmental covariables were used. The environmental covariables with greater impact on the spatial distribution of soil units, in order of importance, were geological, topographic and climatic. Digital classification was based on 142 randomly distributed training areas and 60 sites for validation of results. In the watershed, according to the FAO (2014) WRB Classification System, the following soil groups were identified satisfactorily $(76.67 \%$ for overall accuracy and $71.20 \%$ for the kappa index): Cambisols (35.8\%), Fluvisols (7.16\%), Leptosols (48.25\%), Regosols (8.00\%) and Vertisols 0.79\%). DSM satisfactorily determined the spatial distribution of soil units in the watershed and identified the most influential environmental covariables. 
Index words: digital soil mapping, covariance analysis, soil units, Kappa Index, Mixteca region.

\section{INTRODUCCIÓN}

Un mapa de suelos es una representación a escala de la distribución espacial de las clases de suelo, con base en características físicas, químicas, biológicas y fisiográficas del terreno; identificadas por un símbolo o una leyenda (Jenny, 1941; Lleverino-González et al., 2000). La cartografía de las unidades de suelo y sus propiedades intrínsecas, son información básica en estudios de erosión del suelo (Putman et al., 1988), análisis hidrológico (Mukundan et al., 2010) y modelado ecológico (Tan et al., 2005). De acuerdo con McBratney et al. (2003), en su modelo SCORPAN, el grupo de suelo en un sitio $\left(S_{C}\right)$ está en función de sus propiedades físicas y químicas puntuales $(s)$, el clima del entorno $(c)$, la actividad de los organismos $(o)$, los atributos del relieve $(r)$, el material parental $(p)$, la edad geológica del sitio $(a)$ y la posición espacial del suelo $(n)$. De este modo, el suelo es producto de la interacción de las condiciones ambientales del sitio y entre mayor sea la diversidad de éstas, de una región o cuenca, mayor será la variedad de los suelos. Para representar cartográficamente la diversidad de suelos, tradicionalmente se realizan levantamientos, los cuales requieren de planeación, muestreo intenso, análisis de laboratorio y procesamiento de datos. Este procedimiento es lento y costoso lo cual explica la escasez de mapas de suelos detallados en el ámbito nacional (Abarca y Bernabé, 2010).

El Mapeo Digital de Suelos (MDS o DSM, por sus siglas en ingles Digital Soil Mapping) es una herramienta eficaz para implementar algoritmos complicados, procesar abundantes bases de datos, obtener información de alta precisión y generar cartografía de alta resolución espacial (McBratney et al., 2000; Zhu y Mackay, 2001; McBratney et al., 2003; Dobos et al., 2006). Las técnicas de MDS, con base en observaciones de campo y datos auxiliares, permiten inferir la distribución espacial de los grandes grupos de suelos e identificar variables independientes (covariables) y controles ambientales potenciales de mayor impacto en la formación de los suelos (McBratney et al., 2000; Lagacherie, 2008; Adhikari et al., 2014). El MDS es una técnica ampliamente aceptada para generar cartografía edafológica, a través de covariables ambientales, en áreas donde la información de suelos es escasa. Según Abarca y Bernabé (2010) y Van Zijl et al. (2014), los MDS son útiles para producir rápidamente mapas de suelo con precisión aceptable. Por otro lado, Wahren et al. (2016) establecen que los mapas de suelo, generados a través de MDS, representan de mejor forma la variabilidad espacial de los suelos en una cuenca y son útiles para mejorar la eficiencia de los modelos de simulación hidrológica.

El Mapeo Digital de Suelos (MDS) es una alternativa en estudios donde la información de suelos es mínima o se encuentra a una escala muy pequeña. Por ejemplo, Giasson et al. (2008) utilizaron el MDS para la elaboración de mapas de la distribución de las unidades de suelo, en tres áreas de estudio, con una precisión global entre 61 y 71\% y un Índice Kappa de 36 a 54\%. Por otra parte, Debella-Gilo y Etzelmüller (2009) modelaron, con una precisión global de $27 \%$, la distribución espacial de los grupos de suelos en el sureste de Noruega mediante regresión logística. Adicionalmente, Adhikari et al. (2014) utilizaron el MDS mediante la técnica de árboles de decisión para la obtención de la distribución espacial de los grupos de suelo de acuerdo al sistema de clasificación WRB de la FAO para Dinamarca, teniendo como resultado una precisión global de 60\%. Wahren et al. (2016) emplearon el MDS con el objetivo de comparar la respuesta de un modelo hidrológico a la distribución espacial de las unidades de suelo; donde concluyen que el mapa obtenido a través del MDS representa de mejor forma los atributos espaciales de los suelos, en particular la distribución del contenido de humedad del suelo, debido a que mejoró notablemente los componentes del balance hídrico.

La escasez de mapas detallados de la distribución espacial de las unidades de suelo representa una limitante frecuente en los procesos de tomas de decisiones, el modelado hidrológico y determinación del potencial productivo de un área. Por lo anterior, el objetivo de esta investigación fue obtener la distribución espacial de las unidades de suelo, en la cuenca del río Mixteco, a partir de técnicas de clasificación digital y covariables ambientales relacionadas con los procesos de formación del suelo. 


\section{MATERIALES Y MÉTODOS}

\section{Área de Estudio}

Esta investigación se llevó a cabo en la cuenca del río Mixteco que cubre un área de 655903 ha. En ella confluyen varios ríos como el Salado, Tamazulapa, Huajuapan, Chiquito y el Mixteco, entre otros, de estos, el principal es el Mixteco hasta su desembocadura en la estación hidrométrica Mariscala (Clave 18344). La zona de estudio se ubica en el estado de Oaxaca entre los paralelos $18^{\circ} 02^{\prime} 58.75^{\prime}$ " y $17^{\circ} 03^{\prime} 34.78^{\prime}$ " $\mathrm{N}$ y entre los meridianos $98^{\circ} 16^{\prime} 49.36^{\prime \prime}$ y $97^{\circ} 22^{\prime} 24.94^{\prime \prime} \mathrm{O}$ (Figura 1); con altitudes de 1040 a $3366 \mathrm{~m}$.

La cuenca del río Mixteco se localiza en la provincia fisiográfica denominada Sierra Madre del Sur, específicamente, dentro de tres subprovincias fisiográficas: la Mixteca alta (72.57\%), la cordillera costera del sur (24.29\%) y sierras centrales de Oaxaca (3.14\%). De acuerdo a la carta geológica del INEGI (1980) en la cuenca del río Mixteco las rocas más antiguas datan de la erapaleozoica (17\%) y corresponden a esquistos e ígneas intrusivas ácidas; las rocas de la era mesozoica $(25 \%)$ corresponden básicamente a rocas sedimentarias del tipo calizas, caliza-lutita, caliza-yeso y limolita-arenisca; por último, las rocas de mayor predominancia provienen de la era cenozoica $(58 \%)$ que corresponden principalmente a rocas volcánicas de tipo areniscas, arenisca-conglomerado, ígnea extrusiva ácida, ígnea extrusiva básica, ígnea extrusiva intermedia, ígnea intrusiva intermedia, limolitaarenisca y volcanoclásticas. De acuerdo con el mapa de uso de suelo y vegetación (WWF, 2014) el $45.39 \%$ de la cuenca está cubierta por algún tipo de bosque, un $23.71 \%$ por pastizales y un $9.01 \%$ por agricultura.

\section{Áreas de Entrenamiento}

Con base en los principales cinco grupos de suelo identificados por INEGI (2006), se visitaron 142 sitios distribuidos aleatoriamente (en las principales vías de comunicación de la cuenca) y procurando 28 sitios por grupo de suelo (Figura 1). Del recorrido de campo resultó que 60 sitios correspondieron a suelos del grupo Leptosol, 36 a Fluvisol, 32 a Cambisol, 8 a Regosol y 6 sitios al grupo Vertisol; los cuales fueron usados como áreas de entrenamiento para realizar la clasificación supervisada. Los Cambisoles son suelos con al menos un principio de diferenciación evidente, entre horizontes del subsuelo, por cambios en la estructura, color,

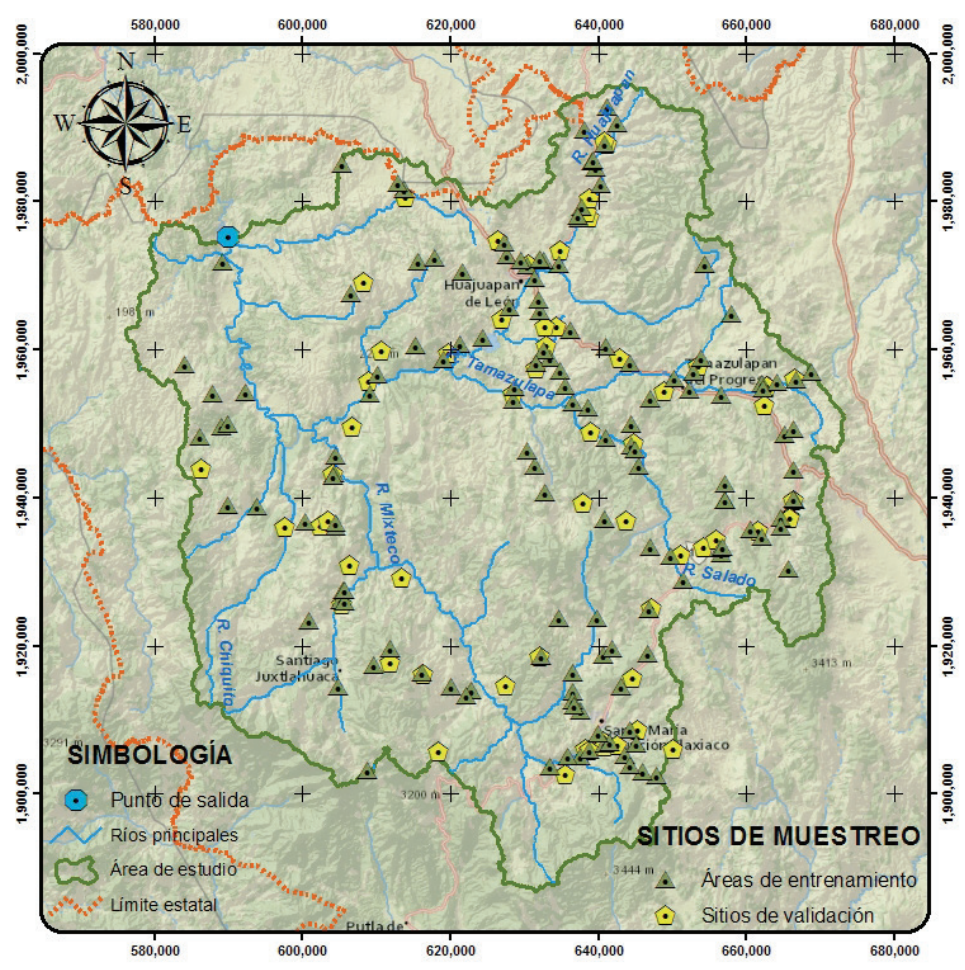

Figura 1. Cuenca del río Mixteco y sitios de muestreo. 
contenido de arcilla o contenido de carbonato (FAO, 2014); para este suelo se identificaron tres unidades del tipo: Crómico, Éutrico y Léptico. Los Fluvisoles son suelos que se desarrollan sobre depósitos aluviales (FAO, 2014); y en el área de estudio se identificaron del tipo Éutrico. Los Leptosoles son suelos que subyacen en lechos rocosos a profundidades menores a $25 \mathrm{~cm}$ (FAO, 2014) y se identificaron las unidades del tipo Lítico y Réndzico. Los Regosoles son suelos producto del depósito de materiales aluviales de tamaño grueso principalmente en el pie de monte $(\mathrm{FAO}, 2014)$ y la unidad dominante fue la Éutrico. Por último, los Vertisoles son suelos arcillosos que tienen un horizonte vértico $(F A O, 2014)$ y la unidad identificada fue del tipo Pélico.

\section{Covariables Ambientales}

Como parte de la metodología se realizó una búsqueda de las covariables ambientales que diversos autores han empleado para el MDS. Pike et al. (2009) identificaron tres grupos de covariables, que son: topográficas, hidrológicas y climáticas. Entre la topográficas, la mayoría de los estudios reportan al Modelo de Elevación Digital (MED) como la fuente principal de información para extraer covariables topográficas y elementos geomorfométricos, entendiendo las primeras como medidas descriptivas de las formas superficiales (es decir, pendiente, orientación de la pendiente, índice topográfico de humedad) y los segundos como entidades espaciales discretas como divisorias de cuencas, abanicos aluviales, red de drenaje (McBratney et al., 2003; Bishop y Minasny, 2006; Pike et al., 2009; Abarca y Bernabé, 2010). Entre las covariables climáticas, algunos autores incluyen la radiación directa, la precipitación y la temperatura (McKenzie y Ryan, 1999; Debella-Gilo y Etzelmüller, 2009; Abarca y Bernabé, 2010). Adicionalmente, a estos grupos de covariables, Adhikari et al. (2014) consideran a las clases de roca, como covariable de tipo geológico.

Para la obtención de las variables topográficas e hidrológicas se utilizó el MDE con $15 \mathrm{~m}$ de resolución del INEGI (2015). Este MDE se corrigió con la función Fill de QGIS $^{1}$ para rellenar vacíos y eliminar imperfecciones en las elevaciones. A partir del MDE corregido se generaron las covariables ambientales.
En el Cuadro 1 se presentan las covariables ambientales seleccionadas para el MDS, en él se muestra su descripción, procedimiento de cálculo, unidad de medida y su factor de formación de acuerdo al modelo SCORPAN.

Las covariables ambientales de Precipitación (PCP) y Temperatura (TEM) se obtuvieron de los registros de las Normales Climatológicas para 78 estaciones climáticas dentro y fuera del área de estudio para el periodo de medición de 1950 a 2000. Se obtuvo un modelo geoestadístico de la PCP a partir de la latitud, longitud, distancia y altura sobre el nivel del mar con un coeficiente de determinación $\left(\mathrm{r}^{2}\right)$ de 0.82 y el modelo geoestadístico de la TEM a partir de la altura sobre el nivel del mar con un coeficiente de determinación de $\left(r^{2}\right)$ de 0.85 .

\section{Análisis Multivariado}

Con el propósito de identificar las covariables ambientales de mayor influencia, en la distribución espacial de los suelos, se realizó un análisis multivariado a través de la técnica de Análisis de Componentes Principales (ACP). Como parte del ACP, se seleccionaron los componentes principales que explicaran al menos el $90 \%$ de la varianza, con base en estos se realizó la clasificación digital supervisada para las unidades de suelo identificadas en campo.

\section{Clasificación Digital}

Con el propósito de agrupar los valores digitales, de los componentes principales seleccionados, se realizó la clasificación digital para conocer la distribución espacial de los grupos de suelos identificadas en campo (Lillesand et al., 2014), de acuerdo al Sistema de Clasificación WRB de la FAO (2014), estos grupos fueron: Cambisoles, Fluvisoles, Leptosoles, Regosoles y Vertisoles. La clasificación digital utilizada en esta investigación fue la supervisada (Macedo-Cruz et al., 2010) y se basó en 142 áreas de entrenamiento representativas de los grupos de suelo indicados.

\section{Evaluación de los Resultados}

Para evaluar la calidad de los mapas generados, se utilizaron los criterios de precisión global y

${ }^{1}$ Quantum GIS Development Team. 2016. Quantum GIS Geographic Information System. Open Source Geospatial Foundation Project. http://qgis.osgeo.org. 
Cuadro 1. Descripción de las covariables ambientales analizadas.

\begin{tabular}{|c|c|c|c|c|c|}
\hline Variable & Significado & Descripción & $\begin{array}{l}\text { Procedimiento o Algoritmo de } \\
\text { cálculo }\end{array}$ & Unidad & $\begin{array}{l}\text { Factor de } \\
\text { formación }\end{array}$ \\
\hline ORA & $\begin{array}{l}\text { Orientación de la } \\
\text { pendiente }\end{array}$ & $\begin{array}{l}\text { Ángulo horizontal, a partir del norte, } \\
\text { en el sentido de las agujas del reloj } \\
\text { (aspecto). Indicador de la incidencia } \\
\text { de la energía solar }\end{array}$ & & Grados & $r$ \\
\hline CUG & Curvatura general & $\begin{array}{l}\text { Tasa de cambio de la pendiente en } \\
\text { el espacio. Indica la concavidad y } \\
\text { convexidad del terreno }\end{array}$ & & Adimensional & $r$ \\
\hline CUH & Curvatura horizontal & $\begin{array}{l}\text { Tasa de cambio horizontal de } \\
\text { la pendiente en la dirección de } \\
\text { máxima pendiente. Determina la } \\
\text { convergencia o divergencia del flujo }\end{array}$ & $\begin{array}{l}\text { SAGA módulo (Terrain Analysis } \\
\text { - Morphometry } \rightarrow \text { Slope, Aspect, } \\
\text { Curvature) }\end{array}$ & Adimensional & $r$ \\
\hline CUV & Curvatura vertical & $\begin{array}{l}\text { Tasa de cambio vertical de la } \\
\text { pendiente en la dirección de } \\
\text { máxima pendiente. Determina la } \\
\text { desaceleración y aceleración relativa } \\
\text { del flujo }\end{array}$ & & Adimensional & $r$ \\
\hline PEN & Pendiente & $\begin{array}{l}\text { Tasa de cambio que expresa la } \\
\text { primera derivada de la elevación } \\
\text { respecto a la superficie horizontal }\end{array}$ & & $\%$ & $r$ \\
\hline IPT & $\begin{array}{l}\text { Índice de posición } \\
\text { topográfica }\end{array}$ & $\begin{array}{l}\text { Índice que caracteriza la topografía } \\
\text { local (geoformas) con respecto a la } \\
\text { posición relativa del terreno }\end{array}$ & $\begin{array}{l}\text { SAGA módulo (Terrain Analysis } \\
\text { - Morphometry } \rightarrow \text { Topographic } \\
\text { Position Index) }\end{array}$ & $\mathrm{m}$ & $r$ \\
\hline ITH & $\begin{array}{l}\text { Índice topográfico de } \\
\text { humedad }\end{array}$ & $\begin{array}{l}\text { Determina la influencia de la } \\
\text { topografía en la concentración de } \\
\text { humedad del suelo y su potencial } \\
\text { para generar escorrentía }\end{array}$ & $\begin{array}{l}\text { SAGA módulo (Terrain Analysis } \\
\text { - Hidrology } \rightarrow \text { Topographic } \\
\text { Wetness Index (TWI)) }\end{array}$ & Adimensional & $r$ \\
\hline LOP & $\begin{array}{l}\text { Longitud de } \\
\text { pendiente }\end{array}$ & $\begin{array}{l}\text { Distancia de máxima pendiente que } \\
\text { recorre el escurrimiento superficial } \\
\text { desde el parteaguas hasta un cauce }\end{array}$ & $\begin{array}{l}\text { SAGA módulo (Terrain Analysis - } \\
\text { Hydrology } \rightarrow \text { Slope Lenght) }\end{array}$ & $\mathrm{m}$ & $r$ \\
\hline IPF & $\begin{array}{l}\text { Índice de potencia de } \\
\text { flujo }\end{array}$ & $\begin{array}{l}\text { Indica la capacidad que acumula una } \\
\text { celda para producir erosión }\end{array}$ & $\begin{array}{l}\text { SAGA módulo (Terrain Analysis } \\
\text { - Hydrology } \rightarrow \text { Stream Power } \\
\text { Index) }\end{array}$ & Adimensional & $r$ \\
\hline ITS & $\begin{array}{l}\text { Índice de transporte } \\
\text { de sedimentos }\end{array}$ & $\begin{array}{l}\text { Caracteriza el efecto de la topografía } \\
\text { sobre las pérdidas de suelo }\end{array}$ & $\begin{array}{l}\text { SAGA módulo (Terrain Analysis - } \\
\text { Hydrology } \rightarrow \text { LS Factor) }\end{array}$ & Adimensional & $r$ \\
\hline DED & Densidad de drenaje & $\begin{array}{l}\text { Define la longitud media de curso } \\
\text { por unidad de superficie }\end{array}$ & $\begin{array}{l}\text { Resultado de la división de la } \\
\text { longitud de las corrientes de } \\
\text { primer orden y área de captación }\end{array}$ & $\mathrm{km} \mathrm{km}^{-2}$ & $p$ \\
\hline PCP & $\begin{array}{l}\text { Precipitación media } \\
\text { anual }\end{array}$ & $\begin{array}{l}\text { Distribución espacial de la lluvia } \\
\text { media anual }\end{array}$ & $\begin{array}{l}\text { Regresión lineal múltiple a partir } \\
\text { de la altitud, latitud, longitud y } \\
\text { distancia al mar }\end{array}$ & $\mathrm{mm}$ & $c$ \\
\hline TEM & $\begin{array}{l}\text { Temperatura media } \\
\text { anual }\end{array}$ & $\begin{array}{l}\text { Distribución espacial de la } \\
\text { temperatura media anual }\end{array}$ & $\begin{array}{l}\text { Regresión lineal a partir de la } \\
\text { altitud }\end{array}$ & ${ }^{\circ} \mathrm{C}$ & $c$ \\
\hline DVO & Distancia a volcanes & $\begin{array}{l}\text { Es la distancia a conos volcánicos } \\
\text { que condiciona el espesor de las } \\
\text { cenizas volcánicas depositadas; } \\
\text { las cuales a través de procesos de } \\
\text { intemperismo químico favorecen } \\
\text { ciertos tipos de suelos presentes en } \\
\text { zonas volcánicas }\end{array}$ & $\begin{array}{l}\text { Distancia euclidiana entre el sitio } \\
\text { y los picos topográficos }\end{array}$ & $\mathrm{m}$ & $p$ \\
\hline
\end{tabular}


el Índice Kappa. El primero representa el grado de dispersión que muestran las propiedades o denominaciones del suelo, alrededor de un perfil típico para una unidad cartográfica (Brown, 1988). El segundo criterio se reconoce como un método estadístico confiable para evaluar la precisión a través de una matriz de error (Congalton y Green, 2008). La matriz de error se compone de números organizados en filas y columnas donde se expresa el número de unidades de la muestra (pixeles, grupos de píxeles, o polígonos) asignadas a una categoría particular en relación con la categoría real (Congalton, 2004). La precisión global e Índice Kappa se obtienen a partir de las siguientes Ecuaciones 1 y 2 :

$$
\begin{aligned}
P & =\frac{\sum_{i=1}^{m} X_{i i}}{N} \\
k & =\frac{N \sum_{i=1}^{m} X_{i i}-\sum_{i=1}^{m} X_{i \Sigma} X_{\Sigma i}}{N^{2}-\sum_{i=1}^{m} X_{i \Sigma} X_{\Sigma i}}
\end{aligned}
$$

dónde: $P$ es la precisión global; $k$ es el coeficiente kappa; $m$ es el número total de unidades; $N$ es el número total de sitios en las $m$ unidades de referencia; $X_{i i}$ son los elementos de la diagonal de la matriz de confusión; $X_{\Sigma i}$ es la suma de los sitios de la unidad $i$ de referencia; $X_{i \Sigma}$ es la suma de los sitios clasificados como la unidad $i$.

\section{RESULTADOS Y DISCUSIÓN}

\section{Selección de Covariables}

A través del ACP se analizó la correlación entre covariables ambientales con el propósito de seleccionar, con fines de clasificación, aquellas covariables con bajas correlaciones, es decir, solo aquellas covariables que aportan información adicional (Ferrero et al., 2002). Para no afectar significativamente los resultados se optó por seleccionar correlaciones inferiores al 55\%. Las covariables que resultaron con valores superiores al $55 \%$ de correlación fueron: CUH $(82.0 \%)$ y CUV (73.0\%), al compararse con CUG, así como ITS $(95.0 \%)$ e IPF (63.0\%), al correlacionarse con PEN. De este modo, para la clasificación de suelos se utilizaron las covariables ORA, CUG, PEN, IPT, ITH, LOP, PCP, TEM, DED y DVO y se eliminaron del análisis CUH, CUV, ITS e IPF por no aportar información relevante.

\section{Jerarquización de Covariables}

Una vez seleccionadas las covariables se analizó el impacto de cada una en la formación de los suelos presentes en la cuenca. Los valores más altos de la diagonal de la matriz de correlaciones (Cuadro 2) corresponden a las covariables ambientales DVO, ORA, PEN y LOP, las cuales acumulan el $60.70 \%$

Cuadro 2. Matriz de covarianza de las covariables ambientales que participan en la formación de suelos, cuenca del río Mixteco,

\begin{tabular}{|c|c|c|c|c|c|c|c|c|c|c|}
\hline $\begin{array}{l}\text { Covariable } \\
\text { ambiental }\end{array}$ & ORA & CUG & PEN & ITH & IPT & LOP & PCP & TEM & DED & DVO \\
\hline ORA & 2916.96 & -1.96 & -30.64 & 0.56 & 10.03 & -57.19 & 31.13 & -38.12 & 18.97 & 30.19 \\
\hline CUG & & 797.37 & 13.86 & 195.53 & -581.42 & -568.10 & -2.50 & -21.17 & -58.98 & -15.52 \\
\hline PEN & & & 1.848 .13 & -35.11 & -911.47 & 363.73 & -371.52 & -30.12 & -254.88 & -449.02 \\
\hline ITH & & & & 334.09 & -281.96 & -279.88 & -1.11 & -29.96 & -90.10 & -7.43 \\
\hline IPT & & & & & 1.472 .00 & 748.86 & 284.84 & 72.02 & 136.36 & 389.21 \\
\hline LOP & & & & & & 1.796 .85 & -22.10 & 50.09 & -104.18 & 97.19 \\
\hline PCP & & & & & & & 1529.59 & 458.02 & 150.15 & 678.86 \\
\hline TEM & & & & & & & & 1563.83 & -165.87 & 423.97 \\
\hline DED & & & & & & & & & 1512.11 & -34.87 \\
\hline DVO & & & & & & & & & & 4574.85 \\
\hline
\end{tabular}
Oaxaca.

$\overline{\mathrm{ORA}}=$ orientación de la pendiente; $\mathrm{CUG}=$ curvatura general; $\mathrm{PEN}=$ pendiente; ITH $=$ índice topográfico de humedad; IPT = índice de posición topográfica; $\mathrm{LOP}=$ longitud de pendiente; $\mathrm{PCP}=$ precipitación media anual; $\mathrm{TEM}=$ temperatura media anual; $\mathrm{DED}=$ densidad de drenaje; $\mathrm{DVO}=$ distancia a volcanes . 
de la varianza total; lo cual implica que estas cuatro covariables son las de mayor impacto en la formación de los suelos. Esto coincide con los resultado obtenidos por Adhikari et al. (2014) quienes concluyen que la geología y las geoformas son determinantes en la distribución espacial de los grupos de suelos, por otra parte Giasson et al. (2008) mencionan a la pendiente como un variable importante en la distribución espacial de los suelos, debido a su participación directa en los procesos dinámicos del acumulación de agua.

\section{Selección de Componentes Principales}

Como parte de ACP, se generaron los autovectores que dan origen a la combinación lineal de los componentes principales y los autovalores correspondientes a cada Componente Principal. De este análisis, resultó que los primeros siete componentes sintetizan el $94.59 \%$ de la variabilidad total de las covariables ambientales seleccionadas para la clasificación digital de los grupos de suelo (Cuadro 3, última fila).

\section{Tipo de Covariables y su Impacto}

De los siete componentes principales seleccionados (Cuadro 4), el CP1 está correlacionado principalmente con DVO (0.955); el CP2 está en función de ORA (0.977); el CP3 depende de IPT (0.851), seguido de CUG (-0.626) y LOP (0.625); el CP4 se relaciona principalmente con PEN (0.754) y LOP (0.667); el CP5 está en función de TEM (0.837); el CP6 depende de DED (0.824) y por último, el CP7 está determinado principalmente por PCP (-0.577) y TEM (0.470). Por lo anterior, se deduce que la distribución espacial de las unidades de suelo está dada en primer lugar por covariables ambientales de tipo geológico, lo cual coincide con Adhikari et al. (2014); seguido por las de tipo topográficas, que gobiernan en gran parte el movimiento de las partículas de suelo; y en tercer lugar por las covariables de tipo climático que proporcionan el medio de transporte de las partículas (Debella-Gilo y Etzelmüller, 2009; Abarca y Bernabé, 2010).

\section{Clasificación de las Unidades de Suelo}

Producto de la clasificación supervisada y con base en el sistema de clasificación WRB de la FAO (2014), para la cuenca del río Mixteco se identificaron ocho unidades de suelo. En la Figura 2 se presentan la distribución espacial de las unidades de suelo clasificadas y en el Cuadro 5 la superficie calculada de cada suelo.

\section{Verificación de las Unidades de Suelo Clasificadas}

La validación de las unidades de suelo, obtenidas con el MDS, se llevó a cabo a través de la matriz de confusión que se muestra en el Cuadro 6. Para la elaboración de la matriz se emplearon 60 sitios, los

Cuadro 3. Autovectores y autovalores de los componentes principales de las covariables ambientales, cuenca del río Mixteco, Oaxaca.

\begin{tabular}{|c|c|c|c|c|c|c|c|c|c|c|}
\hline \multirow{2}{*}{ Autovectores } & \multicolumn{10}{|c|}{ Componente principal } \\
\hline & CP1 & $\mathrm{CP} 2$ & CP3 & $\mathrm{CP} 4$ & CP5 & CP6 & $\mathrm{CP} 7$ & CP8 & CP9 & $\mathrm{CP} 10$ \\
\hline 1 & 0.017 & 0.976 & 0.181 & 0.119 & 0.025 & -0.004 & 0.015 & 0.012 & -0.003 & -0.001 \\
\hline 2 & -0.041 & 0.075 & -0.332 & -0.17 & 0.034 & -0.042 & -0.077 & 0.766 & 0.47 & 0.193 \\
\hline 3 & -0.21 & -0.011 & -0.325 & 0.662 & 0.067 & 0.359 & -0.096 & -0.247 & 0.25 & 0.381 \\
\hline 4 & -0.018 & 0.035 & -0.15 & -0.082 & 0.014 & -0.075 & -0.065 & 0.179 & -0.717 & 0.643 \\
\hline 5 & 0.198 & -0.103 & 0.613 & -0.086 & -0.044 & -0.161 & 0.038 & -0.13 & 0.394 & 0.604 \\
\hline 6 & 0.059 & -0.169 & 0.497 & 0.578 & -0.006 & 0.103 & -0.091 & 0.537 & -0.213 & -0.184 \\
\hline 7 & 0.236 & 0.007 & 0.086 & -0.216 & 0.506 & 0.324 & -0.724 & -0.064 & -0.006 & -0.035 \\
\hline 8 & 0.15 & -0.034 & -0.015 & 0.037 & 0.784 & 0.042 & 0.597 & 0.048 & -0.02 & 0.02 \\
\hline 9 & 0.016 & 0.016 & 0.133 & -0.302 & -0.264 & 0.849 & 0.296 & 0.09 & -0.042 & 0.055 \\
\hline 10 & 0.912 & 0.021 & -0.283 & 0.178 & -0.228 & 0.002 & 0.055 & -0.018 & -0.002 & -0.005 \\
\hline Autovalores & 5015.05 & 2928.55 & 2836.69 & 2393.4 & 1782.21 & 1425.5 & 971.52 & 530.84 & 283.59 & 178.4 \\
\hline Varianza & 27.34 & 15.96 & 15.46 & 13.05 & 9.71 & 7.77 & 5.3 & 2.89 & 1.55 & 0.97 \\
\hline
\end{tabular}


Cuadro 4. Correlación entre los componentes principales y las covariables ambientales, cuenca del río Mixteco, Oaxaca.

\begin{tabular}{lccccccccccc}
\hline \multirow{2}{*}{ Correlación } & \multicolumn{7}{c}{ Componente principal } & & \\
\cline { 2 - 9 } & CP1 & CP2 & CP3 & CP4 & CP5 & CP6 & CP7 & CP8 & CP9 & CP10 \\
\hline ORA & 0.022 & 0.977 & 0.179 & 0.108 & 0.019 & -0.003 & 0.009 & 0.005 & -0.001 & 0.000 \\
CUG & -0.103 & 0.144 & -0.626 & -0.294 & 0.051 & -0.056 & -0.085 & 0.625 & 0.280 & 0.091 \\
PEN & -0.346 & -0.014 & -0.403 & 0.754 & 0.066 & 0.315 & -0.070 & -0.132 & 0.098 & 0.119 \\
ITH & -0.071 & 0.104 & -0.437 & -0.220 & 0.032 & -0.156 & -0.110 & 0.226 & -0.660 & 0.470 \\
IPT & 0.365 & -0.146 & 0.851 & -0.109 & -0.048 & -0.159 & 0.031 & -0.078 & 0.173 & 0.210 \\
LOP & 0.098 & -0.216 & 0.625 & 0.667 & -0.006 & 0.092 & -0.067 & 0.292 & -0.085 & -0.058 \\
PCP & 0.428 & 0.009 & 0.117 & -0.270 & 0.546 & 0.312 & -0.577 & -0.038 & -0.002 & -0.012 \\
TEM & 0.268 & -0.046 & -0.020 & 0.046 & 0.837 & 0.040 & 0.470 & 0.028 & -0.008 & 0.007 \\
DED & 0.030 & 0.023 & 0.182 & -0.379 & -0.287 & 0.824 & 0.237 & 0.054 & -0.018 & 0.019 \\
DVO & 0.955 & 0.016 & -0.223 & 0.129 & -0.142 & 0.001 & 0.025 & -0.006 & 0.000 & -0.001 \\
\hline ORA = orientación de la pendiente; CUG $=$ curvatura general; PEN $=$ pendiente; ITH = índice topográfico de humedad; IPT = índice de posición topográfica;
\end{tabular}

$\mathrm{LOP}=$ longitud de pendiente; $\mathrm{PCP}=$ precipitación media anual; $\mathrm{TEM}=$ temperatura media anual; $\mathrm{DED}=$ densidad de drenaje; $\mathrm{DVO}=$ distancia a volcanes .

cuales corresponden a: 4 sitios para $\mathrm{CMcr}, 7$ para $\mathrm{CMeu}$, 3 para CMlep, 16 para FLeu, 21 para LPli, 4 para LPrz, 3 para RGeu y 2 para VRpe. En la diagonal se muestra el número de sitios validados que concuerdan con la clasificación digital.
Con base en los resultados del Cuadro 6 se observa que la clasificación digital, obtenida a través de el Mapeo Digital Suelos, arrojó una precisión global de $76.67 \%$ y un Índice Kappa de $71.20 \%$, por lo que los resultados de la clasificación se consideran aceptables

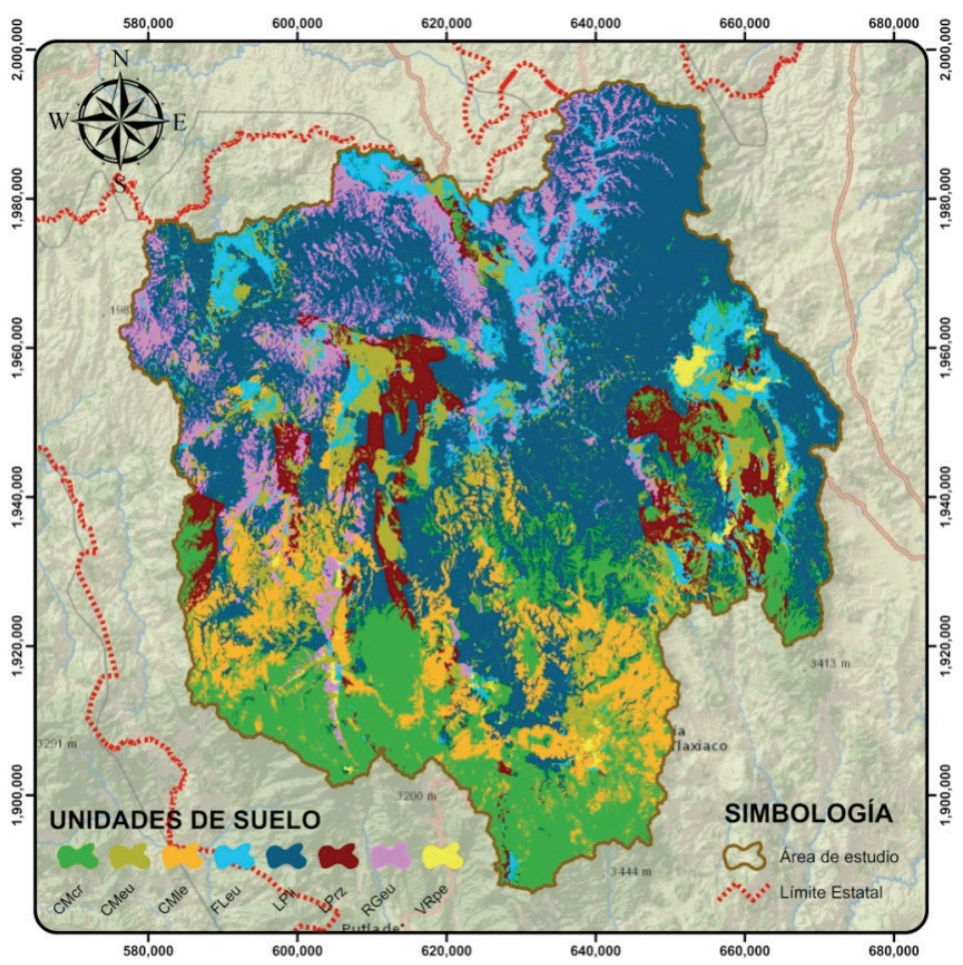

Figura 2. Distribución de las unidades de suelo a partir del MDS, cuenca del río Mixteco, Oaxaca, México. 
Cuadro 5. Unidades de suelos a partir del MDS, cuenca del río Mixteco, Oaxaca, México.

\begin{tabular}{lccrc}
\hline \multirow{2}{*}{ Grupo } & Clasificador & Unidad de Suelo & \multicolumn{2}{c}{ Superficie } \\
\cline { 4 - 5 } Cambisol & Crómico & CMcr & 113464.87 & 17.30 \\
Cambisol & Éutrico & CMeu & 60509.27 & 9.23 \\
Cambisol & Léptico & CMlep & 60782.03 & 9.27 \\
Fluvisol & Éutrico & FLeu & 46979.12 & 7.16 \\
Leptosol & Lítico & LPli & 275591.19 & 42.02 \\
Leptosol & Réndzico & LPrz & 40859.12 & 6.23 \\
Regosol & Éutrico & RGeu & 52537.25 & 8.00 \\
Vertisol & Pélico & VRpe & 5180.13 & 0.79 \\
Total & & & 655903.00 & 100.00 \\
\hline
\end{tabular}

(Bishop et al., 1977). Para las covariables seleccionadas, la precisión global obtenida fue mayor a la presentada por Debella-Gilo y Etzelmüller (2009) quienes usando regresión logística multinomial obtuvieron un valor de $27 \%$. La precisión obtenida en el presente trabajo también mejoró a la reportada por Giasson et al. (2008) quienes obtuvieron, a través de regresión logística, una precisión global de $71 \%$ y un Índice Kappa de 54\%. En este caso, los autores emplearon nueve covariables ambientales y prescindieron las de tipo climático y geológico.

Como se observa en los resultados, el MDE fue la principal fuente de información para obtener covariables de tipo topográfico y climático (a través de regresión lineal múltiple) para inferir la distribución espacial de las unidades de suelo. Actualmente, los MDE tienen como principal fuente de información las curvas de nivel de las cartas topográficas escala 1:50 000 del INEGI, sin embargo, estos datos de elevación tienen el inconveniente de ser generados a través de la interpolación entre curvas. Además de MDE más precisos, para futuros trabajos se sugiere incrementar la intensidad de muestreo e incluir, como parte del análisis, las propiedades físicas y químicas de las principales capas de suelo en cada lugar visitado.

Cuadro 6. Matriz de confusión de la evaluación de la clasificación digital de unidades de suelo, en la cuenca del río Mixteco, Oaxaca, México.

\begin{tabular}{|c|c|c|c|c|c|c|c|c|c|}
\hline \multirow{2}{*}{ Clasificación digital } & \multicolumn{8}{|c|}{ Sitios de verificación } & \multirow{2}{*}{ Total } \\
\hline & $\mathrm{CMcr}$ & Cmeu & CMlep & FLeu & LPli & LPrz & RGeu & VRpe & \\
\hline $\mathrm{CMeu}$ & 0 & 6 & 0 & 1 & 0 & 0 & 0 & 0 & 7 \\
\hline CMlep & 0 & 0 & 2 & 0 & 1 & 0 & 0 & 0 & 3 \\
\hline LPli & 1 & 1 & 3 & 1 & 13 & 0 & 2 & 0 & 21 \\
\hline LPrz & 0 & 0 & 0 & 0 & 0 & 4 & 0 & 0 & 4 \\
\hline RGeu & 0 & 0 & 0 & 1 & 0 & 0 & 2 & 0 & 3 \\
\hline VRpe & 0 & 0 & 0 & 0 & 0 & 0 & 0 & 2 & 2 \\
\hline
\end{tabular}




\section{CONCLUSIONES}

- El Mapeo Digital de Suelos, a través del análisis de componentes principales y covariables ambientales (topográficas, hidrológicas, climáticas y geológicas), resultó satisfactorio (precisión global de 76.67\% y un Índice Kappa de $71.20 \%$ ) para determinar la distribución espacial de las unidades de suelo, según el sistema de clasificación WRB de la FAO (2014), en la cuenca del río Mixteco.

- El Mapeo Digital de Suelos permitió identificar y caracterizar las covariables ambientales de mayor impacto en la distribución espacial de las unidades de suelo otorgando mayor peso, en orden de importancia, a las covariables geológicas, topográficas y climáticas.

\section{LITERATURA CITADA}

Abarca O. y M. A. Bernabé Poveda. 2010. Estimación de la capacidad de uso de las tierras en el estado Aragua, Venezuela, mediante regresión logística multinomial. Agron. Trop. 60: 397-413.

Adhikari, K., B. Minasny, M. B. Greve, and M. H. Greve. 2014. Constructing a soil class map of Denmark based on the FAO legend using digital techniques. Geoderma 214: 101-113.

Bishop, T. F. A. and B. Minasny. 2006. Digital soil-terrain modeling: The predictive potential and uncertainty. pp. 185-213. In: S. Grunwald (ed.). Environmental soil-landscape modeling geographic information technologies and pedometrics. CRC Press. Boca Raton, FL, USA.

Bishop, Y. M., S. E. Fienberg, P. W. Holland, R. J. Light, and F. Mosteller. 1977. Book Review: Discrete multivariate analysis: Theory and practice. pp. 297-306. In: H. Wainer (ed.). Applied psychological measurement. West Publisher Co. Eagan, MN, USA.

Brown, R. B. 1988. Concerning the quality of soil survey. J. Soil Water Conserv. 43: 452-455.

Congalton, R. G. 2004. Putting the map back in map accuracy assessment. pp. 1-11. In: R. S. Lunetta and J. G. Lyon (eds.). Remote sensing and GIS accuracy assessment. CRC Press. Boca Raton, FL, USA.

Congalton, R. G. and K. Green. 2008. Assessing the accuracy of remotely sensed data: Principles and practices. CRC Press. Boca Raton, FL, USA.

Debella-Gilo, M. and B. Etzelmüller. 2009. Spatial prediction of soil classes using digital terrain analysis and multinomial logistic regression modeling integrated in GIS: Examples from Vestfold County, Norway. Catena 77: 8-18.

Dobos, E., F. Carré, T. Hengl, H. I. Reuter, and G. Tóth. 2006. Digital soil mapping as a support to production of functional maps. EUR 22123 EN, Office for Official Publications of the European Communities. Luxembourg.
FAO (Food and Agriculture Organization of the United Nations). 2014. Base referencial mundial del recurso suelo 2014: Sistema internacional de clasificación de suelos para la nomenclatura de suelos y la creación de leyendas de mapas de suelos. Informes sobre Recursos Mundiales de Suelos No. 106. Food and Agriculture Organization of the United Nations. Roma, Italia.

Ferrero, S. B., M. G. Palaci y O. R. Campanella. 2002. Análisis de componentes principales en teledetección. Consideraciones estadísticas para optimizar su interpretación. Rev. Teledetección 17: 43-54.

Giasson, E., S. R. Figueiredo, C. G. Tornquist, and R. T. Clarke. 2008. Digital soil mapping using logistic regression on terrain parameters for several ecological regions in Southern Brazil. pp. 225-232. In: A. E. Hartemink, A. McBratney, and M. L. Mendonca Santos. Digital soil mapping with limited data. Springer. Dordrecht, Netherlands.

INEGI (Instituto Nacional de Estadística y Geografía). 1980. Carta Geológica serie II, escala 1:250 000.(continuo nacional). INEGI. Aguascalientes, México. Sitio Web: http://www.inegi. org.mx/. (Consulta: septiembre 21, 2016).

INEGI (Instituto Nacional de Estadística y Geografía). 2006. Conjunto de datos vectorial edafológico escala 1:250 000 serie II (continuo nacional). INEGI. Aguascalientes, México. Sitio Web: http://www.inegi.org.mx/. (Consulta: septiembre 21, 2016).

INEGI (Instituto Nacional de Estadística y Geografía). 2015. Continuo de elevaciones mexicano. INEGI. Aguascalientes, México. Sitio Web: http://www.inegi.org.mx/. (Consulta: abril 18, 2017).

Jenny, H. 1941. Factors of soil formation: A system of quantitative pedology. McGraw-Hill. New York, NY, USA.

Lagacherie, P. 2008. Digital soil mapping: A state of the art. pp. 3-14. In: A. E. Hartemink, A. McBratney, and M. L. Mendonca Santos. Digital soil mapping with limited data. Springer. Dordrecht, Netherlands.

Lillesand, T. M., R. W. Kiefer, and J. W. Chipman. 2014. Remote sensing and image interpretation. John Wiley and Sons. New York, NY, USA.

Lleverino-González, E., C. A.Ortiz-Solorio y M. C. CastorenaGutiérrez. 2000. La calidad de los mapas de suelos en el ejido Atenco, Estado de México. Terra 18: 103-113.

Macedo-Cruz, A., G. Pajares-Martinsanz y M. Santos-Peñas. 2010. Clasificación no supervisada con imágenes a color de cobertura terrestre. Agrociencia 44: 711-722.

McBratney, A. B., Odeh I.O., Bishop T.F., Dunbar M.S., Shatar T.M. 2000. An overview of pedometric techniques for use in soil survey. Geoderma, 97: 293-327.

McBratney, A. B., M. L. Mendonca Santos, and B. Minasny. 2003. On digital soil mapping. Geoderma 117: 3-52.

McKenzie, N. J. and P. J. Ryan. 1999. Spatial prediction of soil properties using environmental correlation. Geoderma 89: 67-94.

Mukundan, R., D. E. Radcliffe, and L. M. Risse. 2010. Spatial resolution of soil data and channel erosion effects on SWAT model predictions of flow and sediment. J. Soil Water Conserv. 65: 92-104. 
Pike, R. J., I. S. Evans, and T. Hengl. 2009. Geomorphometry: A brief Guide. Dev. Soil Sci. 33: 3-30.

Putman, J., J. Williams, and D. Sawyer. 1988. Using the erosionproductivity impact calculator (EPIC) model to estimate the impact of soil erosion for the 1985 RCA appraisal. J. Soil Water Conserv. 43: 321-326.

Tan, Z., S. Liu, C. A. Johnston, T. R. Loveland, L. L. Tieszen, J. Liu, and R. Kurtz. 2005. Soil organic carbon dynamics as related to land use history in the northwestern Great Plains. Global Biogeochem. Cycles 19: 1-10.

Van Zijl, G. M., D. Bouwer D., J. J. van Tol, and P. A. L. le Roux. 2014. Functional digital soil mapping: A case study from Namarroi, Mozambique. Geoderma 219-220: 155-161.
Wahren, F. T., S. Julich, J. P. Nunes, O. Gonzalez-Pelayo, D. Hawtree, K. H. Feger, and J. J. Keizer. 2016. Combining digital soil mapping and hydrological modeling in a data scarce watershed in north-central Portugal. Geoderma 264: 350-362.

WWF (World Wildlife Fund). 2014. Uso de suelo y vegetación 2010, esc. 1:50000. Servidor cartográfico del Proyecto GEF Mixteca versión 2.0. Sitio Web: http://148.245.245.244:1023/ mixteca/map_default.phtml. (Consulta: septiembre 10, 2016).

Zhu, A. X., D. S. Mackay. 2001. Effects of spatial detail of soil information on watershed modeling. J. Hydrol. 248: 54-77. 